\title{
Double Multiple Streamtube Model for Variable Pitch Vertical Axis Wind Turbines
}

\author{
Conaill E. Soraghan ${ }^{1}$, William E. Leithead ${ }^{2}$, Hong Yue $^{3}$ and Julian Feuchtwang ${ }^{4}$ \\ Dept. of Electronic and Electrical Engineering, University of Strathclyde, Glasgow, G1 1XW, United Kingdom
}

\begin{abstract}
An analytical model based on the double multiple streamtube method is proposed for calculating the rotor performance and aerodynamic blade forces for vertical axis wind turbines (VAWTs) with variable pitch straight blades. An interception method is utilized to determine induction factors which can be used to calculate local blade forces. The model makes allowance for flow expansion, flow curvature, tip loss and dynamic stall. Aerodynamic prediction output from this model is compared with experimental data available in the public domain for validation purposes.
\end{abstract}

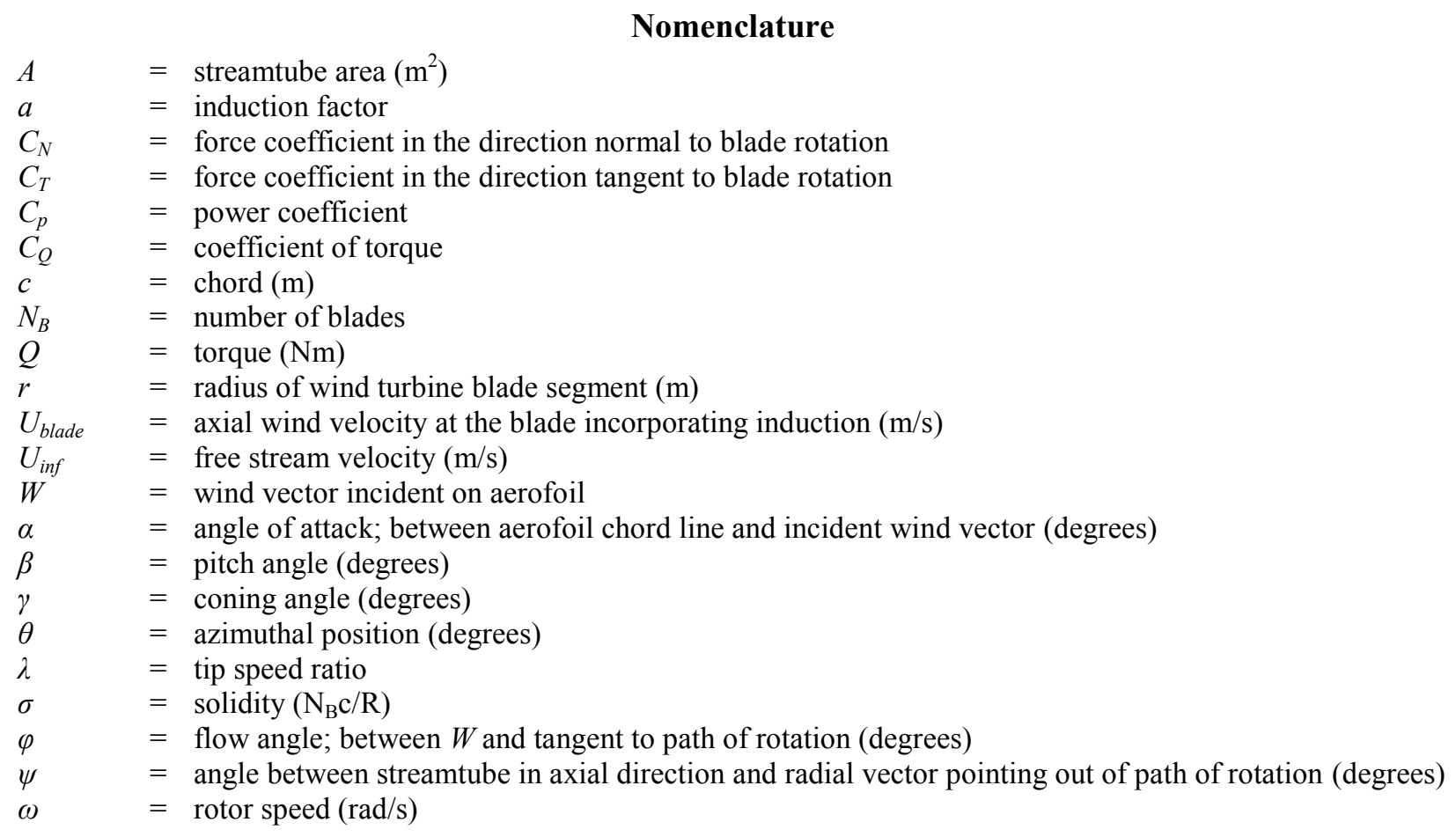

\section{Introduction}

A $\mathrm{S}$ engineers face up to the challenge of designing wind turbines to withstand the harsh environment offshore, there has been a resurgence in vertical axis wind turbine (VAWT) literature ${ }^{[1]}$ and designs ${ }^{[2]}$ due to their simplicity and resilience. Furthermore, designing a floating structure that harnesses energy from the wind is a radically different design challenge compared to that which the industry standard three-blade horizontal axis wind

\footnotetext{
${ }^{1} \mathrm{PhD}$ Candidate, AIAA Student Memeber.

${ }^{2}$ Professor

${ }^{3}$ Lecturer

${ }^{4}$ Research Assisstant
} 
turbine (HAWT) has evolved to serve. VAWTs may prove to be suitable candidates for a floating concept due to their low centre of gravity ${ }^{[3]}$ and stabilising gyroscopic effect ${ }^{[4]}$. Unfortunately, the largescale VAWT concepts that are emerging are mostly stall controlled machines with fixed pitch blades ${ }^{[5-7]}$.

The disadvantages of VAWTs stem from the fact that there is cyclical variation in the angle of attack on the aerofoils as the rotor rotates. As a result, optimal loading cannot be sustained for all azimuthal angles, leading to inherently low aerodynamic efficiency. This is further exacerbated as the blades depart from their optimal lift to drag ratio at most azimuth angles. Additionally, pulsating lift causes significant ripples in the torque and power generated. The ability to pitch the blades as they move around the path of rotation would directly address these disadvantages by sustaining optimal angles of attack for most azimuth angles as the blades rotate ${ }^{[8]}$.

Furthermore, active pitching would improve turbine control. Many of the significant improvements in wind turbine performance over the past three decades have been due to the move towards pitch control. Pitch regulation has not only improved energy capture, but achieved secondary objectives with the most important being the ability to alleviate transient structural loads ${ }^{[9-11]}$. Variable pitching has been considered in the past for VAWTs ${ }^{[12]}$, but active pitch mechanisms were regularly dismissed as too complicated in the context of relatively small turbines. Ingenious passive pitch techniques for VAWTs were investigated ${ }^{[13]}$ but they will never provide the benefits realised from the secondary control objectives of blade pitching that have been developed for HAWT technology.

Before active pitch VAWTs can be built, design tools are required to study how such a turbine would be controlled. It is well established in the literature that variable pitch of VAWT blades can improve aerodynamic efficiency ${ }^{[8,14]}$. However, previous studies focus only on operation at the optimal tip speed ratio and there is a lack of analysis of exploiting pitching for any other control objective. In order to carry out these more general investigations, a double multiple streamtube model has been developed that permits blade pitching. This paper presents the basic representation of the aerodynamics underpinning this model and results are provided for validation purposes. This model utilizes a relatively new approach for calculating induction factors ${ }^{[15]}$ which is referred to as the interception method. It was originally applied to single disk analysis and has not been used before in a double multiple streamtube model. Furthermore, Sharpe's method ${ }^{[16]}$, which captures streamtube expansion, which is critical for accurate modelling of VAWT aerodynamic performance, is adapted to allow for blade pitching.

The novelty of this model lies in its ability to assess the performance of blade pitch regimes. There are almost no aerodynamic prediction models publically available for VAWTs with pitching blades. This work will enable design studies for pitch regulated VAWT controllers .

\section{Aerodynamic Model}

The purpose of an aerodynamic prediction model for wind turbines is to determine the velocity field local to the blades which allows the aerodynamic forces on the blades and power generated by the turbine to be determined. The double multiple streamtube (DMS) method is an extension of blade-element momentum theory ${ }^{[17]}$ that is suitable for modelling VAWTs. It was pioneered by Strickland ${ }^{[18]}$ and amended by Paraschivoiu ${ }^{[19]}$ to represent the turbine as a set of two tandem actuator disks, modelling the up- and downwind passes of the blades. The present model is referred to as StrathDMS. It is developed in MATLAB for convenient use and update. The following sections explain the main features of the aerodynamic prediction code.

\section{A. Geometry}

StrathDMS can predict the aerodynamic performance for H-rotor (vertical blades) and V-rotor (straight blades protruding at an angle $\gamma$ to the vertical) VAWTs with any pitch regime. The swept area is discretised into a mesh by dividing the straight blades into segments and separating the incident wind into independent streamtubes. The convention for angles follows Sharpe's contribution to [16] including a model of streamtube expansion, based on the assumption of straight streamtubes, which has been adapted to permit blade pitching. Figure 1 illustrates the independent straight streamtubes for a choice of 16 azimuthal positions, the set of actuator disks and two crucial angles; $\psi$ is the angle between the streamtube pointing in the downwind direction and the radial vector facing out of the path of rotation; $\theta$ indicates the azimuthal position and is measured anticlockwise from the $\mathrm{x}$ axis pointing downwind. The aerodynamic model permits streamtube expansion and more will be provided on this in section II.C because it depends on the induction factors.

Implicit to this method of analysis is the assumption that there is sufficient slowing of the wind between actuator disks for pressure to recover to the ambient value (half way between two tandem disks). The output of a scheme which determines upstream induction factor $a_{u}$ can be used to calculate an equilibrium wind $U_{e}$ where the pressure has recovered. The pressure recovery assumption allows $U_{e}$ to be used as the input wind speed for a scheme to 
determine downwind induction factor $a_{d}$. The induction factor $\mathrm{a}_{\mathrm{u}}$ is used to define the windspeed at the blade upstream $U_{u}=U_{\text {inf }}\left(1-a_{u}\right)$, and similarly downstream for $U_{d}$. These can be used to determine the areas of the actuator disks, $A_{u}$ and $A_{d}$.

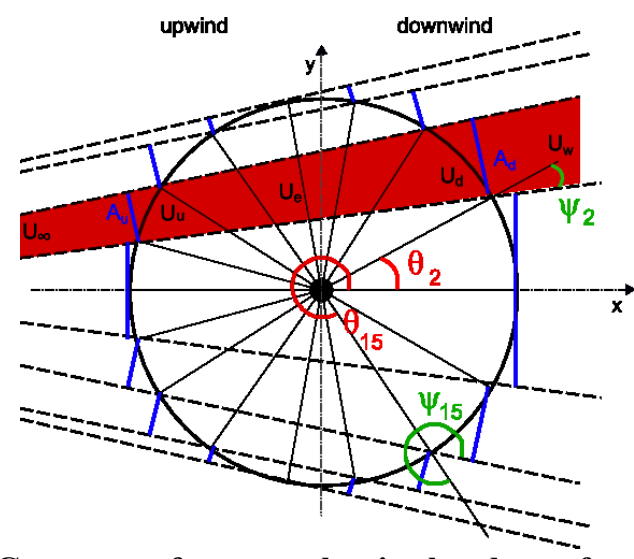

Figure 1. Geometry of streamtubes in the plane of rotation.

The effective wind incident on a VAWT blade, $W$, has two components, the incident wind in the axial direction and wind due to rotation of the blade, which is tangential to the path of rotation. These components and the orthogonal components of $\mathrm{W}$ are illustrated in Fig. 2. $\omega$ is the rotational speed, $r$ is the local radius (which will vary for V-rotors) and $\gamma$ is the coning angle (for V-rotors). $U_{\text {blade }}$ is the wind velocity in the axial direction local to the blade. It incorporates induction and differs up- and downstream according to Eq. (1).

$$
U_{\text {blade }}=\left\{\begin{array}{l}
U_{\text {inf }}\left(1-a_{u}\right)=U_{u}, \text {, upstream } \\
U_{e}\left(1-a_{d}\right)=U_{d}, \text { downstream }
\end{array}\right.
$$

The orthogonal components of $W$ can be used to calculate $\varphi$, the flow angle.

$$
\varphi=\tan ^{-1}\left(\frac{-U_{\text {blade }} \cos \psi \cos \gamma}{U_{\text {blade }} \sin \psi+\omega r}\right)
$$

Once the flow angle is calculated, the pitch angle, $\beta$, is introduced to determine the angle of attack, $\alpha=\varphi-\beta$. This is demonstrated for a blade at $\theta=45^{\circ}$ in Fig.2. In this case $\varphi$ is negative and $\beta$ is positive so $\alpha$ is negative. $\beta$ is introduced to StrathDMS in such a way that it impacts induction factors and hence the degree of streamtube expansion. For all angles in this geometry a clockwise rotation is considered as positive. Therefore positive $\beta$ will cause a nose-out and tail-in pitch as it pitches away from the tangent to rotation. This convention also leads to negative flow angles downstream.

Together, $W$ and $\alpha$ dictate the aerodynamic forces acting on a blade. Figure 2 captures the geometry of the aerodynamic forces acting on a blade at an azimuthal position in the first quadrant. Drag, $D$, is parallel to $W$ and lift, $L$, is perpendicular to $D$, with the sign of $\alpha$ dictating the direction of the lift. Lift and drag coefficients, $C_{L}$ and $C_{D}$, are taken from aerofoil coefficient lookup tables and can be transformed into normal and tangential force coefficients, $C_{N}$ and $C_{T}$, using Eqs. (3).

$$
\begin{aligned}
& C_{N}=C_{L} \cos \varphi+C_{D} \sin \varphi \\
& C_{T}=C_{L} \sin \varphi-C_{D} \cos \varphi
\end{aligned}
$$


Positive normal force, $N$, points in towards the centre of rotation and positive tangential force, $T$, points in the direction of rotation.

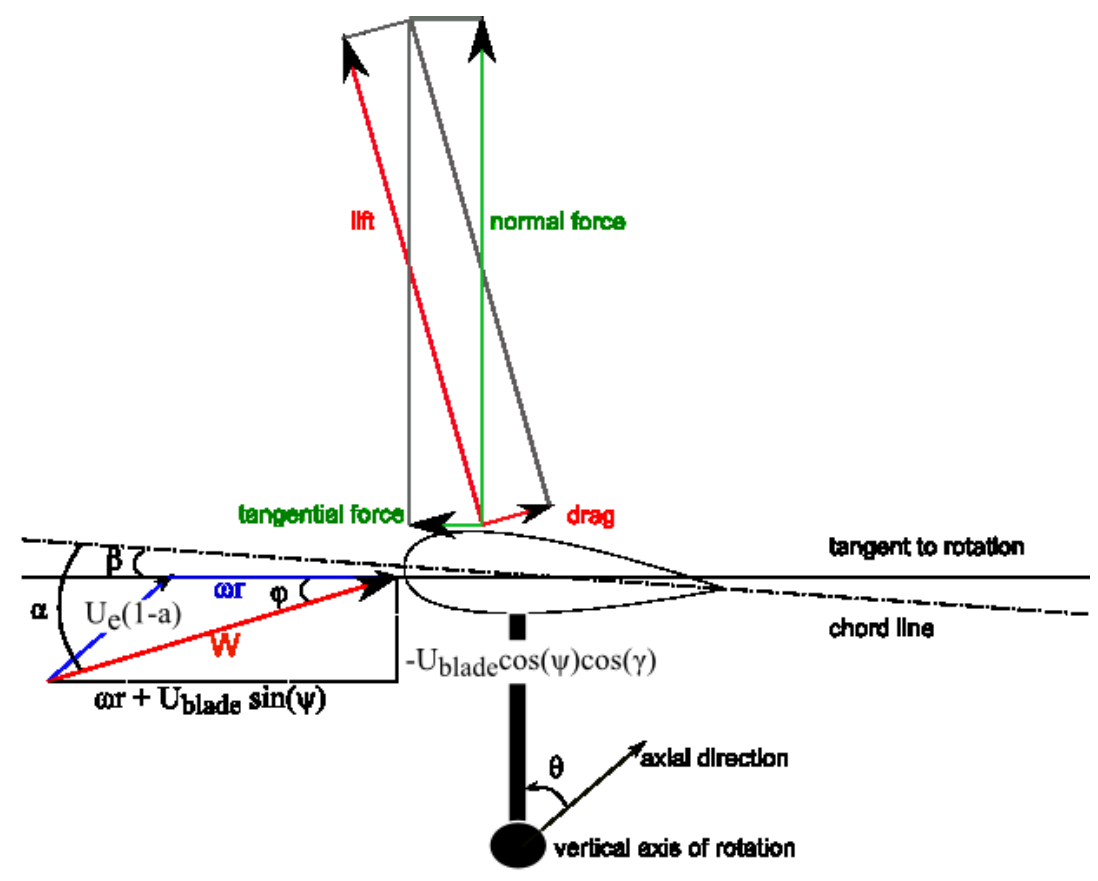

Figure 2. Aerofoil flow variables convention for a blade in the first quadrant at an azimuthal position close to $\mathbf{4 5}^{\circ}$. The blade is downstream so $\varphi$, lift and normal force are all negative. The tail is pitched in so $\beta$ is positive.

\section{B. Interception Method for Determining Induction Factors}

Traditional actuator disk momentum aerodynamic prediction models use an iterative scheme to determine the induction factor, $a$. StrathDMS instead adopts an interception method that was devised by McIntosh, Babinsky and Bertényi ${ }^{[15]}$ for use in a single actuator disk model and applies it to a double actuator disk model. This section explains how it is implemented.

The method is based on the idea that force of the wind on the blades transformed into the axial direction can be determined in two ways simultaneously; using both momentum and blade element considerations. Both of these are functions of $a$ because force on the blades depends on how much energy is absorbed by the blades. Traditional iterative blade element momentum schemes solve for an $a$ where these two calculations for axial force balance. However, the onset of stall and reattachment can lead to multiple solutions and the significant benefit of the interception method of [15] is that it identifies multiple solutions and successfully selects the correct value for $a$.

Force in the axial direction due to momentum considerations is based on actuator disk theory ${ }^{[17]}$ with Glauert's correction.

$$
\operatorname{FxMOM}(a)=\left\{\begin{array}{cc}
4 a(1-a), & a \leq a_{\text {thresh }} \\
1.215 \mathrm{a}+0.485, & a>a_{\text {thresh }}
\end{array}\right.
$$

For $\alpha>\alpha_{\text {thresh }}$ a straight line extending from the standard parabola is used. Constraining the tangent to pass through 1.7 at $a=1$ forces

$$
a_{\text {thresh }}=1-\frac{\sqrt{1.7}}{2}
$$


In order to determine the induction factor, the thrust due to blade element considerations is required. Based on the geometry outlined above which follows [16], the following relationship is employed which is a function of induction factor

$$
\operatorname{FxBE}(a)=\left[\frac{N_{B} c}{2 \pi r}\right] \cdot\left[\frac{W}{U_{\mathrm{inf}}}\right]^{2} \cdot \sec \left(\bmod \left(\psi, \frac{\pi}{2}\right)\right) \cdot\left[-C_{N} \cos \psi \cos \gamma-C_{T} \sin \psi\right]
$$

Where $N_{B}$ is the number of blades, $c$ is chord width, and $W, C_{N}$ and $C_{T}$ are all functions of $a$. Note that for downstream calculations $U_{\text {inf }}$ is replaced by $U_{e}$.

In order to determine an induction factor for each streamtube disk, StrathDMS searches for crossing points of the functions $F x M O M(a)$ and $F x B E(a)$ with an adjustable step size increment in $a$. In the case of multiple crossing points indicating stall detachment or reattachment, the process outlined in [15] is employed to select the appropriate induction factor.

\section{Streamtube Expansion}

Streamtube expansion is the tendency for the flow to expand in the plane of rotation as it passes through the rotor due to conservation of mass and volume as the flow is retarded by the rotor. It is most pronounced in conditions of heavy loading caused by high solidity or high tip speed ratio, $\lambda$, causing the airflow to manoeuver around the turbine rather than through it.

This phenomenon is well understood (section 4.5 .5 of [20]) but, difficulty arises when applying it to momentum models. In general, if flow expansion is to be taken into account, there is a difficulty with the definition of the boundaries between different streamtubes, as the azimuthal position of these boundaries depends on the amount of flow expansion and thereby on the induction factors. As explained in [16], making the approximation that the streamtubes are straight, they can be defined instead initially in terms of the angle $\psi$ between the streamtube and a radial line protruding from the path of rotation. The induction factors can then be calculated before determining the amount of streamtube expansion and the azimuthal positions of the streamtube boundaries.

Once the induction factors have been calculated, the wind speed in the axial direction can be determined. The following expression is used to calculate $U_{e}^{[16]}$

$$
U_{e}=\frac{2 U_{u} U_{d}}{U_{u}+U_{d}}
$$

The area of the streamtube at the pressure recovery point, $A_{e}$, is independent of the induction factors because the straight streamlines pivot about this point for different induction factors

$$
A_{e}=r \cos \left(\bmod \left(\psi, \frac{\pi}{2}\right)\right) \delta_{\psi} \delta_{h}
$$

Where $\delta_{\psi}$ is $2 \pi$ divided by the number of azimuthal positions and $\delta_{\mathrm{h}}$ is the vertical height of a streamtube. Density of air does not vary so conservation of mass flow in the form of Eq. (9) can be used to determine the areas of the streamtubes up- and downstream.

$$
U_{u} A_{u}=U_{e} A_{e}=U_{d} A_{d}
$$

\section{Flow Curvature}

The aerodynamic characteristics of an aerofoil differ for curvilinear and rectilinear flow ${ }^{[21]}$. A symmetrical aerofoil moving in a curved path has the same aerodynamic characteristics as a cambered aerofoil moving in a rectilinear flow field with a virtual angle of incidence. This virtual camber introduces an aerodynamic moment ${ }^{[16]}$ but does not affect induction and energy exchange ${ }^{[22]}$.

Due to the orientation of the virtual camber, an addition to normal force acts toward the centre of rotation. Therefore normal force is increased in the upstream pass and decreased in the downstream pass. Sharpe's analysis in [16] provides an expression for the contribution to normal force as 


$$
C_{N_{f c}}=0.25\left(\frac{d C_{L}}{d \alpha}\right) \frac{c}{r} \lambda
$$

A simplification has been made for StrathDMS; instead of estimating the derivative of $C_{L}$ with respect to $\alpha$, the constant value 5.73 is used. This is a good approximation for low $\alpha$ on the linear section of the $\mathrm{C}_{\mathrm{L}}-\alpha$ curve. A justification for this simplification is that the effects of flow curvature only become significant at large $\lambda$ where $\alpha$ will inevitably be small because the component of wind due to rotation is dominant.

In [22], Simão Ferreira decomposes the bound circulation of a VAWT's blade into steady and unsteady terms. The steady term results from treating the blade's rotation as a constant pitch rate relative to the fixed frame. He concludes that this constant bound circulation (given a constant incident wind speed) does not contribute to wake shedding, and thus has a very small contribution to the induction and energy exchange. However, it does change the blade's loading. Therefore, Sharpe's expression for $\mathrm{C}_{\mathrm{Nfc}}$ is used in eqn. (6) to calculate induction factors, whereas it is not included when quantifying the augmentation to torque due to attaching the blade at the $\mathrm{c} / 4$ point. This is expanded in section section II.F.

\section{E. Aerodynamic Corrections - Tip Loss and Dynamic Stall}

VAWTs have inherently complex aerodynamics due to the continuous changes in angle of attack and blade wake interaction downstream. Two important effects are tip loss and dynamic stall.

Tip loss is the tendency for vorticity to be trailed from the tip of a wind turbine blade, and from any other point where the circulation distribution changes, thereby reducing the blade's effectiveness. StrathDMS applies an adaption of Prandtl's tip loss factor for VAWTs as prescribed in [16]. Tip loss is most pronounced at the ends of the blades but also occurs elsewhere.

Dynamic stall is a complex unsteady aerodynamic phenomenon that can occur when the angle of attack of the wind on an aerofoil changes rapidly. The rate of change of angle of attack as the blade passes the static stall angle has an influence on the manner in which the boundary layer separates or re-attaches. For increasing incidence, the effect is to delay the onset of stall and temporarily increase lift, and for decreasing incidence, it delays flow reattachment. There are several methods for capturing the effects of dynamic stall and a comprehensive review can be found in [23]. StrathDMS adopts the widely used empirical method of Gormont ${ }^{[24]}$.

\section{F. Global Performance Metrics}

Once the aerodynamic forces at each azimuthal position and all streamtube areas have been stored for a specific $\lambda$, the global performance parameters can be calculated. The geometry outlined above leads to the following expression for time averaged torque contribution from each actuator disk

$$
\bar{q}_{i, j}=\frac{1}{2} \rho W_{i, j}{ }^{2}\left[\frac{N c_{i}}{2 \pi r_{i}}\right] \cdot \sec \left(\bmod \left(\psi, \frac{\pi}{2}\right)\right) \cdot A_{i, j} C_{Q_{i, j}}
$$

where $i$ is the blade segment index and $j$ is the azimuthal position index, $A_{i, j}$ is streamtube area and $\rho$ is air density. $\mathrm{C}_{\mathrm{Qi}, \mathrm{j}}$ is the coefficient of torque and this moment coefficient consists of two components as shown in Eq. (12). The first is the tangential force on a blade segment acting at a distance $r$ from the axis of rotation. The second is the normal force acting towards the centre of rotation as a consequence of attaching the blade at the $\mathrm{c} / 2$ point while aerodynamic forces act at the $\mathrm{c} / 4$ point. As was mentioned in section II.D this normal force does not include the contribution from flow curvature given in Eq. (10).

$$
C_{Q_{i, j}}=\left\{\begin{array}{l}
C_{T_{i, j}} r_{i}+\frac{c_{i}}{4} C_{N_{i, j}} \quad \text { upstream } \\
C_{T_{i, j}} r_{i}-\frac{c_{i}}{4} C_{N_{i, j}} \text { downstream }
\end{array}\right.
$$


Total torque provided by the whole turbine, $Q$, is simply the sum of the individual torque contributions as in Eq. (13). Finally, power generated for a specific $\lambda$ is the product of $Q$ and $\omega$.

$$
Q=\sum_{i} \sum_{j} \bar{q}_{i, j}
$$

\section{Validation Results}

In order to validate this analytical model, aerodynamic predictions are presented for a test simulation and subsequently, predicted output is compared with real experimental data published for two different straight blade VAWTs. The novelty of this model lies in its ability to assess the performance of blade pitch regimes therefore both real turbines simulated have pitching blades.

\section{A. Aerodynamic Predicitons for a Test Turbine}

In order to demonstrate StrathDMS output, a test H-rotor has been simulated. The dimensions of the VAWT simulated in this investigation are provided in Table 1. A sinusoidal pitch regime with the objective of increasing the peak aerodynamic efficiency is presented.

\section{Table 1. Dimensions of the VAWT which is simulated in StrathDMS in order to demonstrate the model} output.

\begin{tabular}{|l|l|}
\hline Number of Blades & 3 \\
\hline Radius $(\mathrm{m})$ & 30 \\
\hline Chord $(\mathrm{m})$ & 1.25 \\
\hline Blade Length $(\mathrm{m})$ & 50 \\
\hline Aerofoils & NACA0012 \\
\hline
\end{tabular}

The VAWT selected for this investigation has been specifically designed to operate at peak aerodynamic performance without going into stall. For this to be the case the optimal $\lambda$ must be high enough to maintain low $\varphi$. This constraint ensures that improvements in performance due to pitch do not rely on avoiding stall, but instead must squeeze performance out of the turbine by increasing $|\alpha|$. Figure 3a) illustrates the sinusoidal pitch regime used in this investigation. Figure $3 \mathrm{~b}$ ) demonstrates that the pitch regime is successful in increasing the peak aerodynamic performance. However, this comes at the cost of reducing efficiency at high and low $\lambda$.

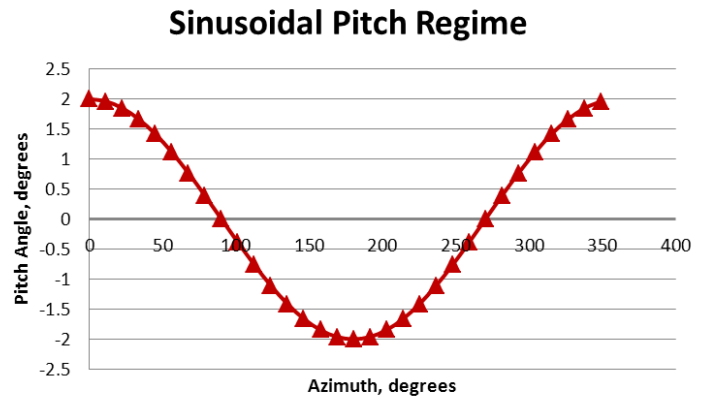

a)

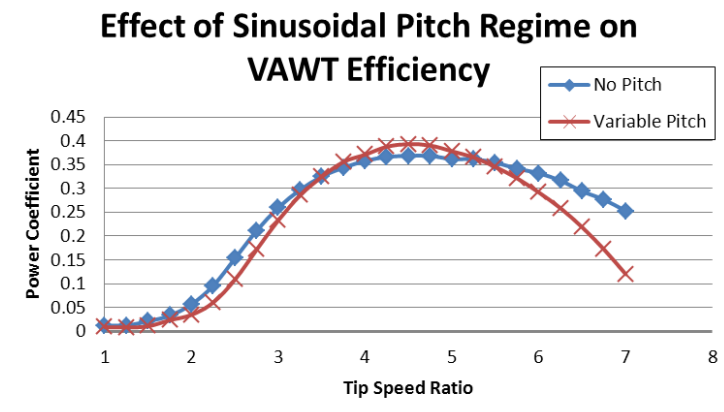

b)

Figure 3. a) The sinusoidal pitch regime designed to improve peak aerodynamic performance. b) Power coefficient against tip speed ratio for the H-rotor test turbine with and without the sinusoidal pitch regime.

Figure 4 demonstrates some important aerodynamic variables which vary with azimuth as predicted by StrathDMS for $\lambda=2.5$. The Reynolds number is $1.7 * 10^{6}$ and the static stall angle is $15^{\circ}$. It can be seen in Fig. 4a) 
that the pitch regime increases $|\alpha|$ both up- and downstream. This is detrimental to performance at this $\lambda$ because the tubine moves further into stall. Induction factor variation with $\theta$ is shown in Fig. 4b). The detrimental effect of the pitch regime is confirmed by Fig. 4c) which demonstrates lower $C_{T}$ with the pitch regime at $0^{\circ}$ and $180^{\circ}$ which correspond to the downstream and upstream positions. Deeper stall has reduced $C_{T}$ which is the driving force of a VAWT. Normal force coefficient is presented in Fig. 4d) and the increase in stall due to the pitch regime has reduced $C_{N}$ up- and downstream.

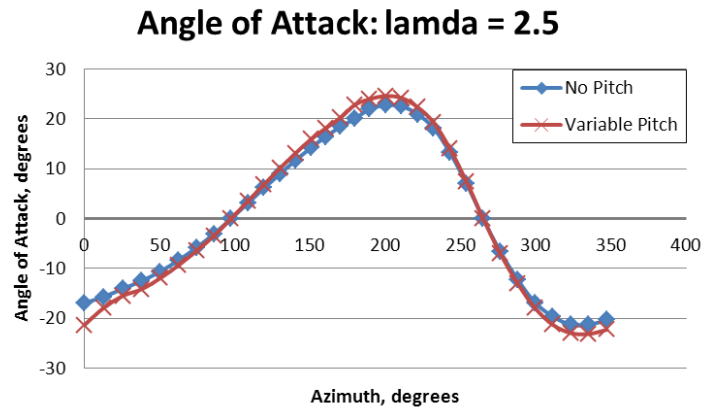

a)
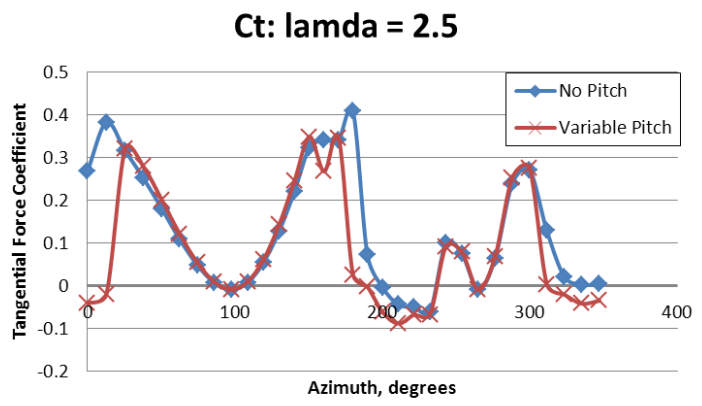

c)

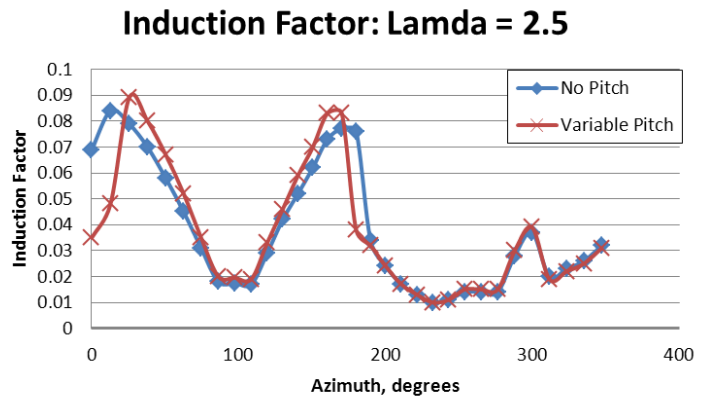

b)

Cn: lamda $=2.5$

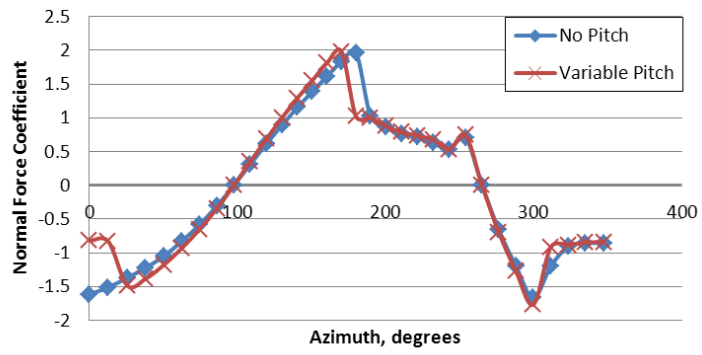

d)

Figure 4. Aerodynamic simulations for the test H-rotor operating at lamda $=2.5$. a) Angle of Attack b) Induction Factor c) Tangential Force Coefficient d) Normal Force Coefficient

Figure 5 demonstrates some important aerodynamic variables which vary with azimuth as predicted by StrathDMS for $\lambda=4.5$. The Reynolds number is $2.7 * 10^{6}$ and the static stall angle is $16^{\circ}$. Figure 5a) shows how angle of attack varies with $\theta$. It is clear that without pitch the angles of attack remain under the static stall angle and the pitch regime increases $|\alpha|$ both up- and downstream. The induction factor is shown in Fig $5 b$ ), there is much smoother variation with $\theta$ at this higher $\lambda$ and the pitch regime causes more induction both up- and downstream. The success of the pitch regime in achieving higher power efficiency at this optimal $\lambda$ is a result of the increase in $C_{T}$ upstream which is demonstrated in Fig 5c). Variation of $C_{N}$ with $\theta$ is presented in Fig $5 \mathrm{~d}$ ) and it is clear that the pitch regime increases the absolute value of normal force. 


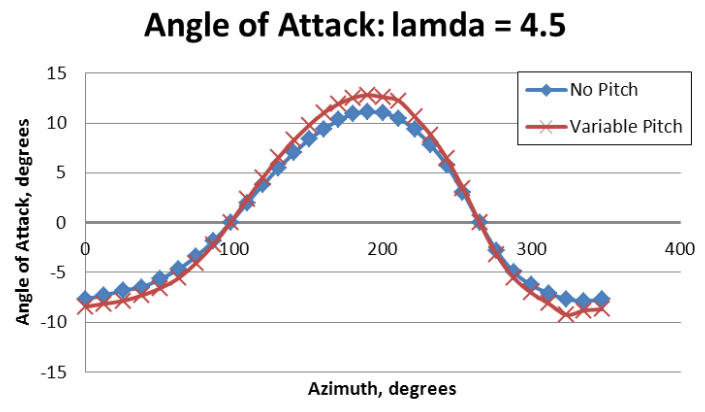

a)

Ct: lamda $=4.5$

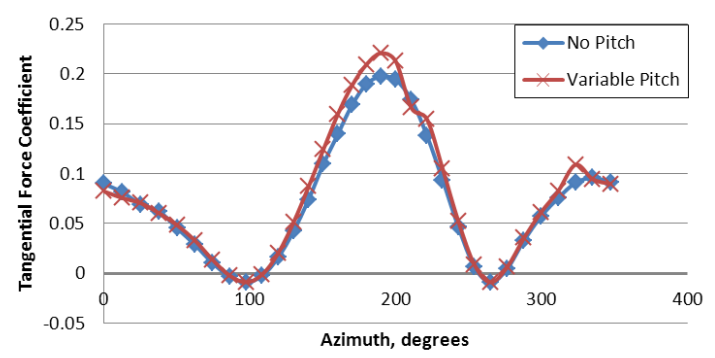

c)

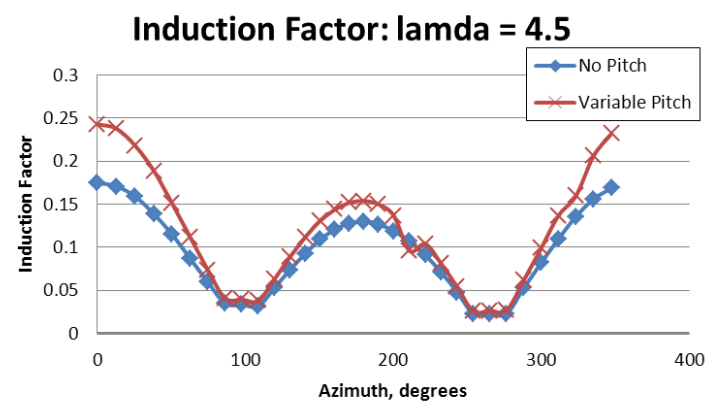

b)

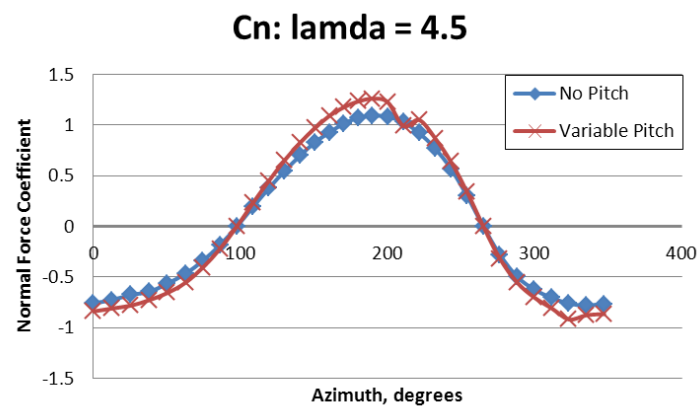

d)

Figure 5. Aerodynamic simulations for the test H-rotor operating at lamda = 4.5. a) Angle of Attack b) Induction Factor c) Tangential Force Coefficient d) Normal Force Coefficient

This investigation highlights that blade pitching can improve VAWT performance, however, pitching can be used for a range of different objectives. The sinusoidal pitch regime in this investigation is successful in achieving an increase in aerodynamic efficiency of the rotor at the optimal $\lambda$ by $5.4 \%$ from 0.37 to 0.39 . Whereas at an offdesign $\lambda$ of $2.5, C_{p}$ drops by $26.7 \%$ from 0.15 to 0.11 . The optimal pith regime applied will depend on the objective to be met and the operating conditions of the turbine. A radically different pitch regime, which aims to decrease $|\alpha|$ and aoid stall, could be designed for lower $\lambda$. Pitching could also be employed to meet structural objectives. VAWTs have notoriously fluctuating power output and forces throughout the structure due to the cyclical angle of attack. Pitching could be used to spread the energy extraction across the whole path of rotation in order to alleviate large force fluctuations. It is the aim of the author to investigate these alternate objectives of VAWT pitching. StrathDMS will be used to examine alternate objectives and how pitch regimes should be modified to suit operation in above and below rated wind speeds.

\section{B. Madsen Turbine}

In 1980 Madsen and Lundgren published results from a field test of a small variable pitch straight bladed VAWT $^{[25]}$. The dimensions of this turbine are provided in Table 2. This VAWT was known as the Voith-Schneider turbine because it used the same mechanism as the similarly named vertical axis propeller used for ship propulsion.

Table 2. Dimensions of the Voith-Schneider turbine built and tested by Madsen and Lundgren.

\begin{tabular}{|l|l|}
\hline Number of Blades & 3 \\
\hline Radius $(\mathrm{m})$ & 1.4 \\
\hline Chord $(\mathrm{m})$ & 0.28 \\
\hline Blade Length $(\mathrm{m})$ & 3.3 \\
\hline Aerofoils & NACA0015 \\
\hline
\end{tabular}


Data are available on power coefficient and reduction in wind speed at the blades. The pitch regime adopted was a basic sinusoidal variation given by Eq. (14) with the convention that $\theta=0$ occurs at the position where the blade moves from downstream to upstream.

$$
\beta(\theta)=3.1+6.6 \sin (\theta)
$$

Figure 6a) is a comparison of experimental results with predicted power coefficients generated by simulating the turbine with StrathDMS. There is spread in the experimental data but it is clear that StrathDMS predicts the maximum $C p$ and optimal $\lambda$ with sufficient accuracy. Parasitic losses from the drag on support structures is modeled using the correction proposed by Vandenberghe in [26] as shown in Eq. (15). The justification is that it was generated for application to a similar small pitching VAWT with equal solidity.

$$
C_{p} \text { loss }=0.0033\left(\lambda^{3}+\lambda\right)
$$

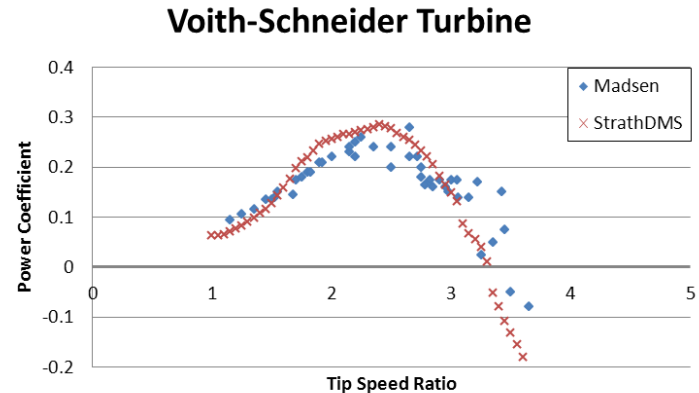

a)

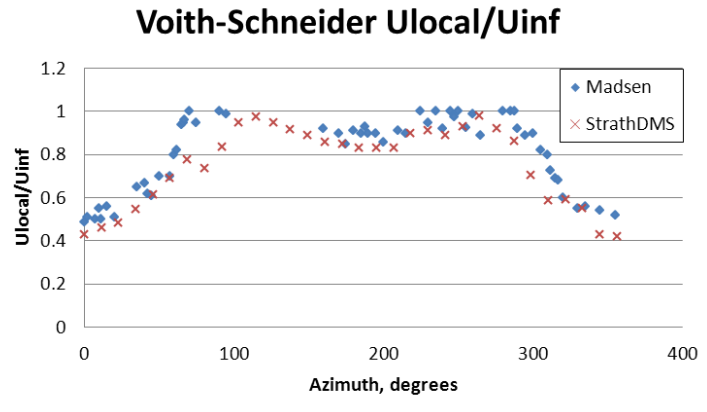

b)

Figure 6. Predictions for the Voith-Schneider VAWT generated by StrathDMS compared with experimental data. a) Power coefficient against tip speed ratio. b) Wind velocity at blade normalised by freestream wind velocity as a function of azimuthal position for a tip speed ratio of 2.8 .

Madsen and Lungren also provided measurements of the azimuthal variation of wind blockage provided by the turbine at $\lambda=2.8$. This is very useful because it can be used to validate induction factors which are the core of the momentum based modelling approach. Figure $6 \mathrm{~b}$ ) is a comparison of experimental data with predictions from StrathDMS for the wind local to the blade in the axial direction normalized by the freestream velocity. This value is labeled Ulocal/Uinf and is equal to1-a. StrathDMS clearly makes good estimations of the induction factors despite the fact that they are consistently slightly higher than the real values. One potential explanation for this is that StrathDMS does not include lateral induction in the $y$ dimension and hence may slightly over-predict how much slowing of the wind there is in the $x$ direction.

\section{Vandenberghe and Dick Turbine}

Vandenberghe and Dick published results from wind tunnel tests for a variable pitch VAWT in $1986^{[27]}$. The dimensions of the turbine are provided in Table 3.

Table 3. Dimensions of the variable pitch VAWT built and tested by Vandenberghe and Dick in 1986.

\begin{tabular}{|l|l|}
\hline Number of Blades & 3 \\
\hline Radius $(\mathrm{m})$ & 0.5 \\
\hline Chord $(\mathrm{m})$ & 0.1 \\
\hline Blade Length $(\mathrm{m})$ & 0.6 \\
\hline Aerofoils & NACA0012 \\
\hline
\end{tabular}


A free vortex model was used by the same authors to determine the optimum pitch regime for this turbine ${ }^{[26]}$. The resulting harmonic pitch control law is given in Eq. (16). As with the Madsen pitch regime, this assumes the convention that $\theta=0$ occurs at the position where the blade moves from downstream to upstream. Equation (16) is the second order optimal pitch regime and to get the first order regime, the expression is truncated at the first order $\theta$ terms.

$$
\begin{aligned}
& \beta(\theta)=2.44-1.95 \cos (\theta)+10.26 \sin (\theta) \\
& -0.52 \cos (2 \theta)-3.40 \sin (2 \theta)
\end{aligned}
$$

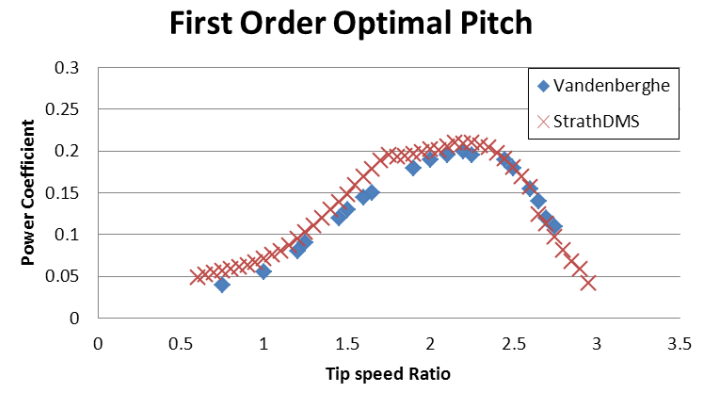

a)

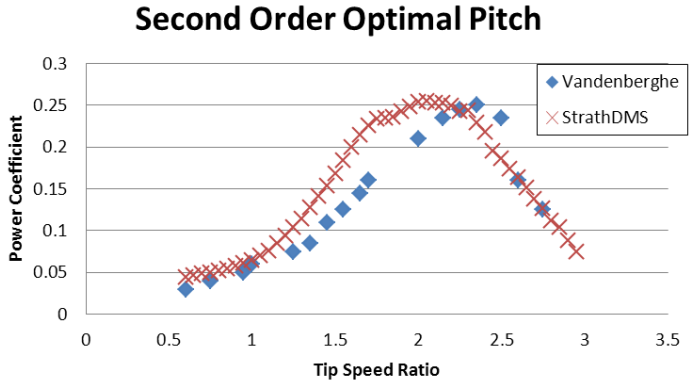

b)

Figure 7. Power coefficient against tip speed ratio for the Vandenberghe and Dick VAWT. Predicted power efficiency from StrathDMS is compared with experimental results. a) first order optimal control regime b) second order optimal control regime.

Figure 7 is a comparison of experimental results with predicted power coefficients generated by simulating the Vandenberghe turbine with StrathDMS. For both a) and b) the correction for structural drag losses given in Eq. (15) is used. The comparison in Fig. 7a) is for the first order pitch regime and it demonstrates that StrathDMS can accurately predict the maximum $C p$, optimal $\lambda$ and performance at all operating regions. The comparison in Fig. $7 \mathrm{~b}$ ) has the second order pitch regime applied and it shows that StrathDMS over-estimates efficiency for the range $1.5<\lambda<2.5$ and the predicted optimal $\lambda$ is slightly lower. Nevertheless, the predicted maximum $C p$ is accurate and the fall off in performance for high lamda is accurate.

\section{Discussion}

This paper provides the outline of a double multiple streamtube analytical tool for prediction of aerodynamic performance of VAWTs with straight pitching blades. The model captures flow curvature effects and involves a geometry with an in-built method for determining streamtube expansion. An interception method is adopted for calculating induction factors which accurately selects the correct induction factor when moving in or out of stall. Two significant aerodynamic effects; tip loss and dynamic stall are modelled by employing standard correction factors. The aerodynamic prediction code is validated against real data and the results illustrate that the model produces accurate predictions for induction factors, blade forces and power output for VAWTs with pitching blades.

Designing a pitch controller for VAWTs is a very complex task because the optimal pitch regime depends on both the operating conditions of the turbine and which objective the controller is attempting to satisfy. From an aerodynamic efficiency point of view, pitching should either increase $|\alpha|$ for pre stall conditions or reduce $|\alpha|$ to avoid stall. However, in terms of the life of the structure it would be beneficial to spread the energy extraction across the whole path of rotation in order to alleviate fluctuating loads. There is an interesting coupling between energy extraction up and downstream so any optimization of pitch regimes, regardless of controller objective, must consider pitching effects at any specific azimuthal location on the performance of the whole turbine. The aerodynamic prediction code presented in this paper will be used to design pitch regimes to suit the range of different control objectives and operating conditions of pitch regulated VAWTs from turbine start up, through Cp-max tracking to above rated energy shedding. 


\section{Acknowledgments}

This work was supported by EPSRC project number EP/G037728/1 for the University of Strathclyde Wind Energy Doctoral Training Centre.

\section{References}

1. Islam, M., D. Ting, and A. Fartaj, Aerodynamic models for Darrieus-type straight-bladed vertical axis wind turbines. Renewable and Sustainable Energy Reviews, 2008. 12(4): p. 1087-1109.

2. Kolios, A., A. Chahardehi, and F. Brennan, Experimental Determination of the Overturning Moment and Net Lateral Force Generated by a Novel Vertical Axis Wind Turbine: Experiment Design Under Load Uncertainty. Society for Experimental Mechanics, 2012. 37: p. 7-14.

3. Vita, L., U. Paulsen, T. Pedersen, H. Madsen and F. Rasmussen, A novel floating offshore wind turbine concept. in EWEC. 2009. Marseille: EWEC Proceedings Online.

4. Crozier, A., Design and Dynamic Modeling of the Support Structure for a 10 MW Offshore Wind Turbine, Masters Thesis in Department of Energy and Process Engineering. 2011, Norwegian University of Science and Technology: Trondheim.

5. MODEC. Floating Wind and Current Hybrid Power Generation. 2013; Available from: http://www.modec.com/fps/skwid/pdf/skwid.pdf.[accessed on 15 May 2013]

6. INFLOW. Industrialization setup of a Floating Offshore Wind Turbine. 2012; Available from: http://www.inflow-fp7.eu/index.html. [accessed on 20 May 2013]

7. SEATWIRL. Sea Twirl Technology Specifications. 2011; Available from: http://www.seatwirl.com/SeaTwirl.pdf. [accessed on 12 April 2013]

8. Staelens, Y., F. Saeed, and I. Paraschivoiu. A straight-bladed variable-pitch VAWT concept for improved power generation. in 41 st Aerospace Sciences Meeting and Exhibit. 2003. Nevada: AIAA.

9. Leithead, W., S. De la Salle, and D. Reardon, Role and objectives of control for wind turbines. Generation, Transmission and Distribution, IEE Proceedings, 1991. 138(2): p. 135-148.

10. Leithead, W., S. De la Salle, D. Reardon and M. Grimble, Wind turbine modelling and control. in International Conference on Control. 1991. Edinburgh: IET.

11. Bossanyi, E., Wind turbine control for load reduction. Wind Energy, 2003. 6(3): p. 229-244.

12. Kirke, B. and L. Lazauskas, Enhancing the performance if a vertical axis wind turbine using a simple variable pitch system. Wind Engineering, 1991. 15(4): p. 187-195.

13. Kirke, B.K., Evaluation of self-starting vertical axis wind turbines for stand-alone applications, PhD Thesis in Department of Engineering. 1998, Griffith University: Southport.

14. Paraschivoiu, I., O. Trifu, and F. Saeed, H-Darrieus wind turbine with blade pitch control. International Journal of Rotating Machinery, 2009. 2009: p. 7 pages.

15. McIntosh, S., H. Babinsky, and T. Bertényi, Convergence Failure and Stall Hysteresis in Actuator-Disk Momentum Models Applied to Vertical Axis Wind Turbines. Journal of Solar Energy Engineering, 2009. 131: p. 034502.

16. $\quad$ Freris, L., Wind energy conversion systems. 1990, New York: Prentice Hall.

17. Burton, T., N. Jenkins, D. Sharpe and E. Bossanyi, Wind energy handbook. 2011, West Sussex: Wiley.

18. Strickland, J., Darrieus turbine: a performance prediction model using multiple streamtubes. 1975, Technical Report for Sandia Nat. Labs.: New Mexico.

19. Paraschivoiu, I. Double-multiple streamtube model for Darrieus wind turbines. in Second DOE/NASA Wind Turbines Dynamics Workshop 1981. Clevland, Ohio.

20. Vries, O., Fluid dynamic aspects of wind energy conversion. 1979, Technical Report for The Advisory Group for Aerospace Research and Development: Amsterdam.

21. Migliore, P. and W. Wolfe. Some effects of flow curvature on the performance of Darrieus wind turbines. in 17th Aerospace Sciences Meeting. 1979. New Orleans: AIAA.

22. Ferreira, C., The near wake of the VAWT: $2 D$ and $3 D$ views of the VAWT aerodynamics, PhD Thesis in Dept. of Aerospace Engineering. 2009, Delft University of Technology: Delft.

23. Larsen, J.W., S.R.K. Nielsen, and S. Krenk, Dynamic stall model for wind turbine airfoils. Journal of Fluids and Structures, 2007. 23(7): p. 959-982.

24. Gormont, R., A mathematical model of unsteady aerodynamics and radial flow for application to helicopter rotors. 1973, Technical Report for The Army Air Mobility Research and Development Laboratory: Pennsylvania.

25. Madsen, H. and K. Lundgren, The Voith-Schneider Wind Turbine: Some Theoretical and Experimental Results on the Aerodynamics of the Voith-Schneider Wind Turbine. 1980, Technical Report for The Institute of Industrial Constructions and Energy Technology: Aalborg.

26. Vandenberghe, D. and E. Dick, Optimum pitch control for vertical axis wind turbines. Wind Engineering, 1987. 11(5): p. 237-247.

27. Vandenbereghe, D. and E. Dick, $A$ theoretical and experimental investigation into the straight bladed vertical axis wind turbine with second order harmonic pitch control. Wind Engineering, 1986. 10: p. 122-138. 